GLASNIK MATEMATIČKI

Vol. 39(59)(2004), $21-26$

\title{
A REMARK ON CENTRALIZERS IN SEMIPRIME RINGS
}

\author{
IRENA KosI-UlBL \\ University of Maribor, Slovenia
}

\begin{abstract}
The purpose of this paper is to prove the following result: Let $m \geq 1, n \geq 1$ be fixed integers and let $R$ be a $(m+n+2)$ !-torsion free semiprime ring with the identity element. Suppose there exists an additive mapping $T: R \rightarrow R$, such that $T\left(x^{m+n+1}\right)=x^{m} T(x) x^{n}$ holds for all $x \in R$. In this case $T$ is a centralizer.
\end{abstract}

This research has been motivated by the work of Vukman [7]. Throughout, $R$ will represent an associative ring with center $Z(R)$. A ring $R$ is $n$ torsion free, where $n$ is an integer, in case $n x=0, x \in R$ implies $x=0$. As usual the commutator $x y-y x$ will be denoted by $[x, y]$. We shall use basis commutator identities $[x y, z]=[x, z] y+x[y, z]$ and $[x, y z]=[x, y] z+y[x, z]$. Recall that $R$ is prime if $a R b=(0)$ implies $a=0$ or $b=0$, and is semiprime if $a R a=(0)$ implies $a=0$. An additive mapping $D: R \rightarrow R$, where $R$ is an arbitrary ring, is called a derivation in case $D(x y)=D(x) y+x D(y)$ holds for all pairs $x, y \in R$ and is called a Jordan derivation in case $D\left(x^{2}\right)=D(x) x+x D(x)$ holds for all $x \in R$. Obviously, any derivation is a Jordan derivation. The converse is in general not true. Herstein [5] proved that any Jordan derivation on a 2-torsion free prime ring is a derivation. A brief proof of Herstein theorem can be found in [3]. Cusack [4] has extended Herstein theorem to 2torsion free semiprime rings (see also [1] for an alternative proof). An additive mapping $T: R \rightarrow R$ is called a left (right) centralizer in case $T(x y)=T(x) y$ $(T(x y)=x T(y))$ holds for all $x, y \in R$. We follow Zalar [8] and call $T$ a centralizer in case $T$ is both left and right centralizer. In case $R$ has the identity element $T: R \rightarrow R$ is left (right) centralizer iff $T$ is of the form $T(x)=a x$ $(T(x)=x a)$ for some fixed element $a \in R$. An additive mapping $T: R \rightarrow R$ is called a left (right) Jordan centralizer in case $T\left(x^{2}\right)=T(x) x\left(T\left(x^{2}\right)=x T(x)\right)$

2000 Mathematics Subject Classification. 16N60, 39B05.

Key words and phrases. Prime ring, semiprime ring, left (right) centralizer, left (right) Jordan centralizer, centralizer. 
holds for all $x \in R$. Following ideas from [1] Zalar [8] has proved that any left (right) Jordan centralizer on a 2-torsion free semiprime ring is a left (right) centralizer. Recently Vukman [6] has proved that in case $T: R \rightarrow R$ is an additive mapping, where $R$ is a 2-torsion free semiprime ring, which satisfies the identity $2 T\left(x^{2}\right)=T(x) x+x T(x)$ for all $x \in R$, then $T$ is a centralizer. In [7] Vukman set the following conjecture:

Let $R$ be a semiprime ring with suitable torsion restrictions. Suppose there exists an additive mapping $T: R \rightarrow R$, such that $T\left(x^{m+n+1}\right)=$ $x^{m} T(x) x^{n}$ holds for all $x \in R$, where $m \geq 1, n \geq 1$ are some integers. In this case $T$ is a centralizer.

The result below gives an affirmative answer to the question above in case $R$ has the identity element.

Theorem 1. Let $m \geq 1, n \geq 1$ be fixed integers and let $R$ be a $(m+n+2)$ !-torsion free semiprime ring with the identity element. Suppose there exists an additive mapping $T: R \rightarrow R$, such that $T\left(x^{m+n+1}\right)=$ $x^{m} T(x) x^{n}$ holds for all $x \in R$. In this case $T$ is a centralizer.

Let us see the background of the conjecture and the theorem above. An additive mapping $D: R \rightarrow R$, where $R$ is an arbitrary ring, is called Jordan triple derivation, in case $D(x y x)=D(x) y x+x D(y) x+x y D(x)$ holds for all pairs $x, y \in R$. One can easily prove that any Jordan derivation on an arbitrary ring is a Jordan triple derivation (see [3]). Brešar [2] has proved that any Jordan triple derivation on a 2-torsion free semiprime ring is a derivation. This result inspired Vukman [7] to prove the following result: Let $T: R \rightarrow R$ be an additive mapping, where $R$ is a 2 -torsion free semiprime ring. Suppose that

$$
T(x y x)=x T(y) x
$$

holds for all $x \in R$. In this case $T$ is a centralizer. For $y=x$ the identity (1) reduces to

$$
T\left(x^{3}\right)=x T(x) x, \quad x \in R .
$$

The question arises whether the identity (2) on a 2-torsion free semiprime ring implies that $T$ is a centralizer. Vukman [7] proved that the answer to this question is affirmative in case $R$ has the identity element. The identity (2) leads to the conjecture above.

Proof of Theorem 1. We have the relation

$$
T\left(x^{m+n+1}\right)=x^{m} T(x) x^{n}, \quad x \in R .
$$


Replacing in the above relation $x+e$ for $x$, where $e$ denotes the identity element, we obtain

$$
\begin{aligned}
& \sum_{i=0}^{m+n+1}\left(\begin{array}{c}
m+n+1 \\
i
\end{array}\right) T\left(x^{m+n+1-i}\right)= \\
& =\left(\sum_{i=0}^{m}\left(\begin{array}{c}
m \\
i
\end{array}\right) x^{m-i}\right)(T(x)+a)\left(\sum_{i=0}^{n}\left(\begin{array}{c}
n \\
i
\end{array}\right) x^{n-i}\right), \quad x \in R
\end{aligned}
$$

where $a$ stands for $T(e)$. Using (3) and rearranging the equation (4) in sense of collecting together terms involving equal number of factors of $e$ we obtain:

$$
\begin{aligned}
& \left(\begin{array}{c}
m+n+1 \\
1
\end{array}\right) T\left(x^{m+n} e\right)-\left(\begin{array}{c}
m \\
0
\end{array}\right)\left(\begin{array}{c}
n \\
1
\end{array}\right) x^{m} T(x) x^{n-1} e-\left(\begin{array}{c}
m \\
0
\end{array}\right)\left(\begin{array}{c}
n \\
0
\end{array}\right) x^{m} a x^{n} \\
& -\left(\begin{array}{c}
m \\
1
\end{array}\right)\left(\begin{array}{c}
n \\
0
\end{array}\right) x^{m-1} T(x) x^{n} e \\
& +\left(\begin{array}{c}
m+n+1 \\
2
\end{array}\right) T\left(x^{m+n-1} e^{2}\right)-\left(\begin{array}{c}
m \\
0
\end{array}\right)\left(\begin{array}{l}
n \\
2
\end{array}\right) x^{m} T(x) x^{n-2} e^{2} \\
& -\left(\begin{array}{c}
m \\
0
\end{array}\right)\left(\begin{array}{c}
n \\
1
\end{array}\right) x^{m} a x^{n-1} e-\left(\begin{array}{c}
m \\
1
\end{array}\right)\left(\begin{array}{c}
n \\
1
\end{array}\right) x^{m-1} T(x) x^{n-1} e^{2} \\
& -\left(\begin{array}{c}
m \\
1
\end{array}\right)\left(\begin{array}{c}
n \\
0
\end{array}\right) x^{m-1} a x^{n} e-\left(\begin{array}{c}
m \\
2
\end{array}\right)\left(\begin{array}{c}
n \\
0
\end{array}\right) x^{m-2} T(x) x^{n} e^{2} \\
& +\cdots+ \\
& +\left(\begin{array}{c}
m+n+1 \\
m+n
\end{array}\right) T\left(x e^{m+n}\right)-\left(\begin{array}{c}
m \\
m-1
\end{array}\right)\left(\begin{array}{c}
n \\
n
\end{array}\right) x a e^{m+n-1} \\
& -\left(\begin{array}{c}
m \\
m
\end{array}\right)\left(\begin{array}{c}
n \\
n
\end{array}\right) T(x) e^{m+n}-\left(\begin{array}{c}
m \\
m
\end{array}\right)\left(\begin{array}{c}
n \\
n-1
\end{array}\right) a x e^{m+n-1}=0, \quad x \in R,
\end{aligned}
$$

or shortly

$$
\sum_{i=1}^{m+n} f_{i}(x, e)=0, \quad x \in R,
$$

where $f_{i}(x, e)$ stands for the expression of terms involving $i$ factors of $e$.

Replacing $e$ by $2 e, 3 e, \ldots,(m+n) e$ in turn in the equation (5), and expressing the resulting system of $m+n$ homogeneous equations, we see that the coefficient matrix of the system is a van der Monde matrix

$$
\left[\begin{array}{cccc}
1 & 1 & \cdots & 1 \\
2 & 2^{2} & \cdots & 2^{m+n} \\
\vdots & \vdots & \vdots & \vdots \\
m+n & (m+n)^{2} & \cdots & (m+n)^{m+n}
\end{array}\right]
$$


Since the determinant of the matrix is different from zero, it follows that the system has only a trivial solution.

In particular,

$$
\begin{aligned}
f_{m+n-1}(x, e)= & \left(\begin{array}{c}
m+n+1 \\
m+n-1
\end{array}\right) T\left(x^{2}\right)-\left(\begin{array}{c}
m \\
m-2
\end{array}\right) x^{2} a-\left(\begin{array}{c}
n \\
n-2
\end{array}\right) a x^{2} \\
& -\left(\begin{array}{c}
m \\
m-1
\end{array}\right) x T(x)-\left(\begin{array}{c}
n \\
n-1
\end{array}\right) T(x) x \\
& -\left(\begin{array}{c}
m \\
m-1
\end{array}\right)\left(\begin{array}{c}
n \\
n-1
\end{array}\right) x a x \\
= & 0, \quad x \in R
\end{aligned}
$$

and

$$
\begin{aligned}
f_{m+n}(x, e)= & \left(\begin{array}{c}
m+n+1 \\
m+n
\end{array}\right) T(x)-\left(\begin{array}{c}
m \\
m-1
\end{array}\right) x a-\left(\begin{array}{c}
n \\
n-1
\end{array}\right) a x \\
& -\left(\begin{array}{c}
m \\
m
\end{array}\right)\left(\begin{array}{c}
n \\
n
\end{array}\right) T(x) \\
= & 0, \quad x \in R .
\end{aligned}
$$

Since $R$ is a $(m+n+2)$ !-torsion free ring, the above equations reduce to

$$
\begin{aligned}
(m+n+1)(m+n) T\left(x^{2}\right)= & m(m-1) x^{2} a+n(n-1) a x^{2}+2 \text { mnxax } \\
& +2 m x T(x)+2 n T(x) x, \quad x \in R
\end{aligned}
$$

and

$$
(m+n) T(x)=\operatorname{mxa}+\operatorname{nax}, \quad x \in R,
$$

respectively.

According to (7) one obtains the relation

$$
(m+n) T\left(x^{2}\right)=m x^{2} a+n a x^{2}, \quad x \in R .
$$

Using the above connection one can replace the expression $(m+n) T\left(x^{2}\right)$ with $m x^{2} a+n a x^{2}$ in the relation (6). Thus we have

$$
\begin{aligned}
(m+n+1)\left(m x^{2} a+n a x^{2}\right)= & m(m-1) x^{2} a+n(n-1) a x^{2} \\
& +2 m n x a x+2 m x T(x)+2 n T(x) x
\end{aligned}
$$

From the above relation we obtain

$$
\begin{aligned}
& (2 m+m n) x^{2} a+(2 n+m n) a x^{2}-2 m n x a x \\
& -2 m x T(x)-2 n T(x) x=0, \quad x \in R .
\end{aligned}
$$

Rearranging the above relation gives

$$
\begin{aligned}
& 2 m\left(x^{2} a-x T(x)\right)+2 n\left(a x^{2}-T(x) x\right) \\
& +m n\left(x^{2} a+a x^{2}-2 x a x\right)=0, \quad x \in R .
\end{aligned}
$$


Left and right multiplication of the relation (7) by $x$ gives

$$
(m+n) x T(x)=m x^{2} a+n x a x, \quad x \in R
$$

and

$$
(m+n) T(x) x=\operatorname{mxax}+n a x^{2}, \quad x \in R,
$$

respectively.

Multiplication of the relation (9) by $(m+n) e$ gives

$$
2 m\left((m+n) x^{2} a-(m+n) x T(x)\right)+2 n\left((m+n) a x^{2}-(m+n) T(x) x\right)
$$

$$
+m n(m+n)\left(x^{2} a+a x^{2}-2 x a x\right)=0, \quad x \in R .
$$

Using (10) and (11) in the relation (12) one obtains

$$
\begin{aligned}
0= & 2 m\left((m+n) x^{2} a-m x^{2} a-n x a x\right)+2 n\left((m+n) a x^{2}-m x a x-n a x^{2}\right) \\
& +m n(m+n)\left(x^{2} a+a x^{2}-2 x a x\right) \\
= & \left(2 m(m+n)-2 m^{2}+m n(m+n)\right) x^{2} a \\
& +\left(2 n(m+n)-2 n^{2}+m n(m+n)\right) a x^{2}-(4 m n+2 m n(m+n)) x a x \\
= & m n(m+n+2) x^{2} a+m n(m+n+2) a x^{2} \\
& -2 m n(m+n+2) x a x, \quad x \in R .
\end{aligned}
$$

Since $R$ is a $(m+n+2)$ !-torsion free ring we obtain

$$
x^{2} a+a x^{2}-2 x a x=0, \quad x \in R .
$$

The above relation can be written in the form

$$
[[a, x], x]=0, \quad x \in R .
$$

The rest of the proof goes through in the same way as in the end of the proof of Theorem 2 in [7], but we proceed for the sake of completeness. Putting $x+y$ for $x$ in the above relation we obtain

$$
[[a, x], y]+[[a, y], x]=0, \quad x, y \in R .
$$

Putting $x y$ for $y$ in relation (15) we obtain because of (14) and (15):

$$
\begin{aligned}
0 & =[[a, x], x y]+[[a, x y], x] \\
& =[[a, x], x] y+x[[a, x], y]+[[a, x] y+x[a, y], x] \\
& =x[[a, x], y]+[[a, x], x] y+[a, x][y, x]+x[[a, y], x] \\
& =[a, x][y, x], \quad x, y \in R .
\end{aligned}
$$

Thus we have

$$
[a, x][y, x]=0, \quad x, y \in R .
$$

The substitution $y a$ for $y$ in the above relation gives

$$
[a, x] y[a, x]=0, \quad x, y \in R .
$$


Let us point out that so far we have not used the assumption that $R$ is semiprime. Since $R$ is semiprime, it follows from the relation (16) that $[a, x]=0, x \in R$. In other words, $a \in Z(R)$, which reduces the relation $(7)$ to $T(x)=a x, x \in R$. The proof of the theorem is complete.

ACKNowledgements.

I would like to thank to Professor Joso Vukman for helpful suggestions.

\section{REFERENCES}

[1] M. Brešar, Jordan derivations on semiprime rings, Proc. Amer., Math. Soc. 104 (1988), 1003-1006.

[2] M. Brešar, Jordan Mappings of semiprime rings, J. Algebra 127 (1989), 218-228.

[3] M. Brešar and J. Vukman, Jordan derivations on prime rings, Bull. Austral. Math. Soc. 3 (1988), 321-322.

[4] J. Cusack, Jordan derivations on rings, Proc. Amer. Math. Soc. 53 (1975), 1104-1110.

[5] I. N. Herstein, Jordan derivations of prime rings, Proc. Amer. Math. Soc. 8 (1957), 1104-1110.

[6] J. Vukman, An identity related to centralizers in semiprime rings, Comment. Math. Univ. Carolinae 40 (1999), 447-456.

[7] J. Vukman, Centralizers of semiprime rings, Comment. Math. Univ. Carolinae, 42 (2001), 237-245.

[8] B. Zalar, On centralizers of semiprime rings, Comment. Math. Univ. Carolinae 32 (1991), 609-614.

Department of Mathematics

University of Maribor

PEF, Koroška c. 160, 2000 Maribor

Slovenia

E-mail: irena.kosi@uni-mb.si

Received: 03.02.2003

Revised: 19.03 .2003 